\title{
PHENOBARBITAL CONFERS ITS DIVERSE EFFECTS BY ACTIVATING THE ORPHAN NUCLEAR RECEPTOR CAR
}

\author{
Susumu Kodama and Masahiko Negishi \\ Pharmacogenetics Section, Laboratory of Reproductive and Developmental Toxi- \\ cology, National Institute of Environmental Health Sciences, National Institutes of \\ Health, Research Triangle Park, North Carolina, USA
}

In the early 1960s, phenobarbital (PB) was shown to induce hepatic drug metabolism and the induction was implicated in the molecular mechanism of drug tolerance development. Since then, it has become evident that PB not only induces drug metabolism, but also triggers pleiotropic effects on liver function, such as cell growth and communication, proliferation of the endoplasmic reticulum, tumor promotion, glucose metabolism, steroid/ thyroid hormone metabolism, and bile acid synthesis. Upon activation by $P B$ and numerous $P B$-type inducers, the nuclear receptor CAR mediates those pleiotropic actions by regulating various hepatic genes, utilizing multiple regulatory mechanisms.

Key Words: Liver; CAR; Nuclear receptors; Hepatic metabolism; Gluconeogenesis; Hepatocellular carcinoma; Gene transcription; Cytochrome P450.

In grappling with the difficult process of accepting Dr. David Kupfer's death, I (M.N.) could not help but reminisce about the earliest encounters with phenobarbital (PB) that I had as a new graduate student at the Institute of Protein Research, Osaka University, 1967. Under the supervision of Professor Tsuneo Omura, I ran the first technique to conduct my project: how to inject PB into rats through tail vein. The project was to investigate the synthesis of cytochrome b5 in rat livers, in which PB was used to induce the cytochrome (Negishi and Omura, 1970). These experiments introduced me to the concept of drug induction. It was also around this time that I ran across an article by Dr. Alan Conney entitled, "Pharmacological implications of microsomal enzyme induction" (Conney, 1967). I still clearly remember the significant impact this article made on the direction of my research: PB was to be on my mind forever. Looking back these 38 years, I realize that PB has always been a subject of my research. Therefore, you can well imagine the deep sense of satisfaction/fulfillment I felt when it was observed that mice lacking the nuclear receptor CAR gene will sleep longer than normal mice following $\mathrm{PB}$ administration (Swales and Negishi, 2004). Here we describe a short history of how CAR emerged as a coordinate factor that regulates diverse liver functions (Fig. 1). This review is by no means a comprehensive coverage of CAR research.

Address correspondence to Dr. Masahiko Negishi, Pharmacogenetics Section, Laboratory of Reproductive and Developmental Toxicology, National Institute of Environmental Health Sciences, Research Triangle Park, NC 27709, USA; Fax: 919-541-0696; E-mail: negishi@niehs.nih.gov 


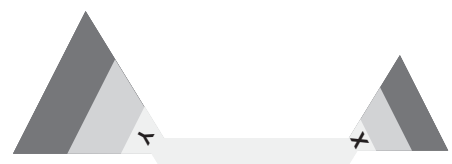

PB

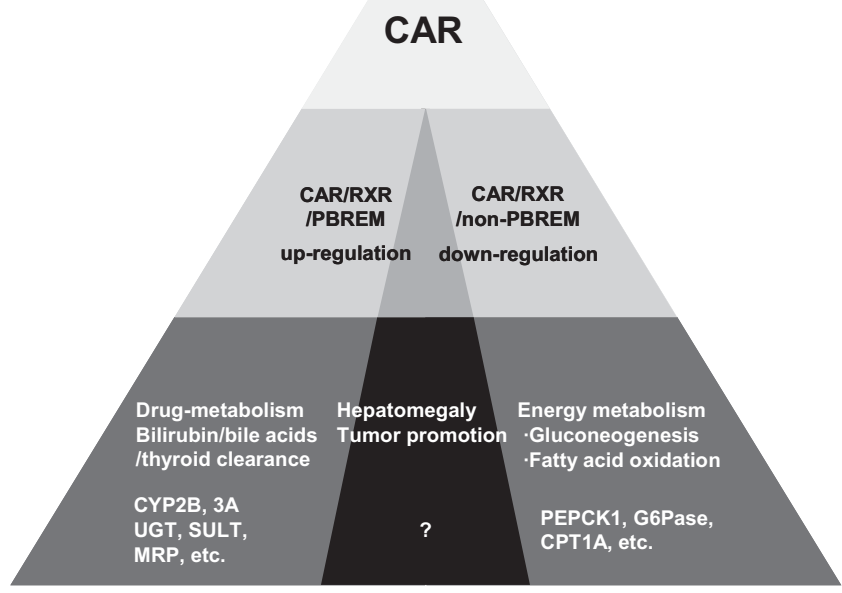

Figure $1 \mathrm{~PB}$ activates CAR to diversify its effects. CAR activation results in the coordinated up- or down-regulation of numerous hepatic genes, altering various liver functions from drug metabolism, to bilirubin secretion, to gluconeogenesis, to fatty acid oxidation, and to tumor promotion (Ueda et al., 2002). Because CAR regulates half of the hepatic genes that were up- or down-regulated by $\mathrm{PB}$, there must be additional factors (X and $\mathrm{Y}$ ) that coordinate the CAR-independent PB actions.

\section{PBREM}

The first PB-inducible gene was cloned and sequenced in the early 1980s (Mizukami et al., 1983); however, it wasn't until the mid 1990s when the molecular mechanism of PB induction began to be understood. Anderson and his associates used rat primary hepatocytes to identify a 176-bp PB response sequence in the far upstream region of the CYP2B2 promoter (Trottier et al., 1995). Subsequently, Paavo Honkakoski delineated this $\mathrm{PB}$ response activity to the 51-bp sequence in the mouse Cyp2b10 gene, termed the PB-responsive enhancer module or PBREM (Honkakoski et al., 1998). Finally, the Omiecinski group, producing a transgenic mouse bearing the mutated sequence, showed that PB-induced transcription of the $C Y P 2 B$ gene requires the presence of the PBREM (Ramsden et al., 1999). In addition to PB, the PBREM is activated in response to numerous PB-type inducers; its sequence is conserved in the mouse, rat, and human $C Y P 2 B$ genes (Honkakoski et al., 1998; Sueyoshi, 1999). The nuclear receptor binding motif DR4 is the key element confering PB response activity to the PBREM (Honkakoski et al., 1998). DR4 motifs are found in CAR-regulated genes such as CYP3A, CYP2C, and UGT1A1 (Swales and Negishi, 2004; Sueyoshi and Negishi, 2001). The identification of the PBREM provided us with the foundation for further elucidating the mechanism of PB induction. 
Nuclear receptor CAR. Most importantly, PBREM allowed researchers to identify a PB-activating nuclear factor. Using transient transfection assays with a PBREMreporter gene, Paavo Honkakoski screened various nuclear receptors for their ability to activate the PBREM in HepG2 cells. Igor Zelko used DNA (PBREM) affinity chromatography to purify a nuclear protein from mouse liver nuclear extracts that bound to the PBREM in response to $\mathrm{PB}$. Their work resulted in the characterization of the nuclear receptor CAR (NR1I3) as the key regulator of PBREM and CYP2B gene (Honkakoski et al., 1998). Subsequently, Takeshi Kawamoto demonstrated that CAR is retained in the cytoplasm in mouse livers and translocates into the nucleus to activate the transcription of the $C y p 2 b$ gene in response to PB treatment (Kawamoto, 1999). Finally, the laboratory of David Moore used $\mathrm{Car}^{-1-}$ mice to confirm that the presence of CAR is essential in the induction of the Cyp $2 b$ gene by PB (Wei et al., 2000). Nearly half a decade has passed since PB induction was first reported. The PB-CAR pathway is now established as the accepted induction mechanism of the $C Y P 2 B$ gene.

Consequences of CAR activation. A recent cDNA microarray analysis revealed that CAR up-regulates a set of genes that encode CYP2B, CYP2C, CYP3A, NADPH-cytochrome P450 reductase, sulfotransferases, glucuronosyltransferases, and glutathione S-transferases (Ueda et al., 2002). In addition to these phase I and II drug-metabolizing enzymes, CAR also up-regulates drug transporters such as Mrp2 and Mrp4 (Kast et al., 2002; Assem et al., 2004). Thus, PB activation of CAR diversifies its ability to induce various enzymes and transporters, increasing the hepatic activities of drug metabolism and secretion. Given the fact that numerous therapeutic drugs and xenobiotics can activate CAR, this receptor constitutes a central defense mechanism against their toxicity and carcinogenicity (Yamamoto et al., 2003). The presence of CAR shortens the duration of PB-induced sleeping time, highlighting the roles that CAR plays against chemical toxicity and/or drug efficacy (Swales and Negishi, 2004).

These same enzymes and transporters are also involved in the metabolism and secretion of endobiotics, such as steroid and thyroid hormones, bilirubin, and bile acids. Therefore, activation of CAR can exert protective or adverse effect on liver functions. Bilirubin is the catabolic byproduct of heme derived from $\beta$-globin and cytochromes and is the most toxic of all endobiotic byproducts. Increased accumulation of bilirubin can cause hyperbilirubinemia. PB is used to treat mild forms of hyperbilirubinemia, such as Gilbert disease, since it induces UDP-bilirubin glucuronosyltransferase (UGT1A1), the rate-limiting enzyme of bilirubin metabolism. Junko Sugatani found that CAR regulates PB-induced transcription of the UGT1AI gene and identified the CAR-responsive enhancer element gtNR1 (Sugatani et al., 2002). In addition, CAR also mediates PB induction of glutathione S-transferase A1/A2 and the transporters OATP2 and MRP2 to increase bilirubin clearance (Huang et al., 2003).

Bile acids are the major products of cholesterol catabolism and, acting as physiological detergents, help intestinal absorption of lipophilic nutrients. The level of bile acids is regulated by the balance of synthesis and elimination; cytochrome P450 7A (CYP7A) is the rate-limiting enzyme of bile acid synthesis, while phase I and II enzymes and transporters play a major role in the elimination (Sinal et al., 2000; Eloranta and Kullak-Ublick, 2005). While CAR does not regulate the expression of CYP7A, the receptor appears to play a role in bile acid elimination by inducing enzymes and transporters (Guo et al., 2003; Zhang et al., 2004). Excess accumulation of bile acids, particularly those of high hydrophobicity, such as lithocholic acid, impairs liver function and causes intrahepatic cholestasis. Treatment with the potent CAR activator TCPOBOP protects against intraheptic cholestasis in the 
$\mathrm{Car}^{+/+}$but not in $\mathrm{Car}^{-/-}$mice (Zhang et al., 2004). PB has been used for decades to treat pruritis associated with intrahepatic cholestasis (Jenkins and Boothby, 2002); CAR may play a role in the response to this treatment.

Chronic treatment with $\mathrm{PB}$ and $\mathrm{PB}-$ like inducers is known to promote thyroid hypertrophy in humans and both hypertrophy and tumors in rats (Hiasa et al., 1982; Curran and DeGroot, 1991; Diwan et al., 1996). Levels of thyroid hormone (TH) are determined by a balance of synthesis, metabolism, and secretion. Thyroid stimulating hormone (TSH) regulates synthesis of inactive tetraiodothyronine (T4) in the thyroid gland. T4 is converted to the bioactive triiodothyronine (T3) by deiodenases in the peripheral target tissues, such as the liver and kidney (Bianco et al., 2002). Phase II enzymes sulfate or glucuronidate T3 and T4 to promote their biliary and urinary excretion (Visser, 1990). Recently, two groups have reported that treatment with PB or TCPOBOP decreases the levels of total serum T4 20 to 50\% in $\mathrm{Car}^{+/+}$but not $\mathrm{Car}^{-/-}$mice (Maglich et al., 2004; Qatanani et al., 2005). CAR-dependent induction of conjugation enzymes such as UGT1A1 and SULT1A1 was then proposed as the basis for this decrease. Given these findings, Qatanani et al. suggested that the decrease of total T4 levels increases TSH, causing thyroid hypotrophy. However, since serum levels of total T3 were not affected by treatments and those of free $\mathrm{T} 4$ were not determined, implicating these conjugation enzymes in the specific elimination of T4 may be problematic. A major flaw of the two studies is the fact that they did not show whether the serum level of free T3, the clinical criterion of TH function, is affected by CAR. So, the question still remains whether the decrease of total T4 has any implication in TH activity and function.

In humans, chronic treatment with PB-like inducer phenytoin is also reported to decrease levels of total serum T4, promoting thyroid hypertrophy (Hegedus et al., 1985, Curran and DeGroot, 1991, Wang, et al., 2004). However, it is not known whether this decrease is caused by the augmentation of metabolism and/or excretion of $\mathrm{TH}$ or the attenuation of TH synthesis. Because human SULT1A1 appears to sulfate both T3 and T4 (Li et al., 2001), it is problematic to suggest that this enzyme is responsible for specific elimination of T4. In addition, human SULT1A1 is not induced by PB (Hempel et al., 2004). Thus, the role of CAR in the PB-induced thyroid diseases remains elusive, but an important question for future investigations.

CAR, where is it driving us? $P B$ activates CAR to induce phase I and II enzymes and transporters, increasing metabolism and secretion of both xenobiotics and endobiotics. So far, what has been established for the mechanism of PB induction is the direct binding of CAR to the PB-responsive enhancer elements such as PBREM and DR4, activating the transcription of genes that encode these enzymes. Now we are coming to realize that there is also a type of CAR regulation that cannot be explained by this mechanism. For example, Susumu Kodama investigated the molecular mechanism of PBinduced repression of gluconeogenic enzymes, such as phosphoenolpyruvate carboxykinase 1 (PEPCK1), in which CAR, acting as co-repressor, regulates the insulin response transcription factor FoxO1 (Kodama et al., 2004). The repression of FoxO1 by CAR provides a molecular basis for understanding a long-standing question of drug-insulin cross-talk.

Chronic PB treatment reduced plasma glucose levels in diabetic patients (Lahtela et al., 1985). Hepatic gluconeogenic enzymes, such as PEPCK1 and glucose 6-phosphatase (G6Pase), are repressed in PB-treated rats and mice (Argaud et al., 1991; Manenti et al., 1987). Moreover, it was found that CAR regulates the PB-induced repression of PEPCK1 (Ueda et al., 2002), in which the receptor directly binds and represses FoxO1 (Kodama et al., 2004). FoxO1 is a $70 \mathrm{~K}$ protein and a member of the Forkhead box "Other" (FoxO) 
proteins. The Forkhead transcription factor family is characterized by a highly conserved "winged-helix" DNA-binding motif, known as a Forkhead box. FoxO proteins contain a unique insert of five amino acids within the $\alpha$-helix 3 of the Forkhead box that defines their sequence-specific DNA binding (Barthel et al., 2005). FoxO proteins directly bind to a consensus sequence [(T/C)(G/A)AAACAA] known as the insulin response sequence (IRS) in the promoter region of gluconeogenic genes, such as PEPCK1 and G6Pase, and activate their transcription (Schmoll et al., 2000; Yeagley et al., 2001; Nakae et al., 2001; Puigserver et al., 2003). The mechanism of insulin regulation of these genes is as follows: Insulin binds to the insulin receptor at the cell membrane to activate phosphatidylinsitol 3 kinase (PI3K). Subsequently, PI3K activates Akt to phosphorylate FoxO1. Once phosphorylated, FoxO1 is excluded from the nucleus, resulting in the repression of IRS-bearing genes (Biggs et al., 1999; Rena et al., 2001). In addition to the nuclear exclusion, it is also suggested that the phosphorylated FoxO1 undergoes ubiquitination and proteasomal degradation (Matsuzaki et al., 2003). The phosphorylation-dependent nuclear exclusion and degradation of FoxO1 is a major pathway of insulin repression of gluconeogenesis. $\mathrm{CAR}$ is now found to repress gluconeogenesis by regulating FoxO1.

Using CAR as bait, Susumu Kodama identified FoxO1 as a CAR-binding protein and elucidated the cross-talk mechanism of FoxO1 and CAR (Fig. 2, Kodama et al., 2004). GST pull-down as well as mammalian two hybrid assays were performed to confirm direct binding of CAR to FoxO1. Co-expression of CAR repressed the FoxO1mediated trans-activation of the IRS in HepG2 cells, similar to the repression by insulin. Activation of Akt by insulin phosphorylates FoxO1, which is removed from the nucleus, resulting in the repression of IRS-bearing genes such as PEPCKI and G6Pase. CAR forms a complex with the un-phosphorylated FoxO1. Because this CAR-FoxO1 complex

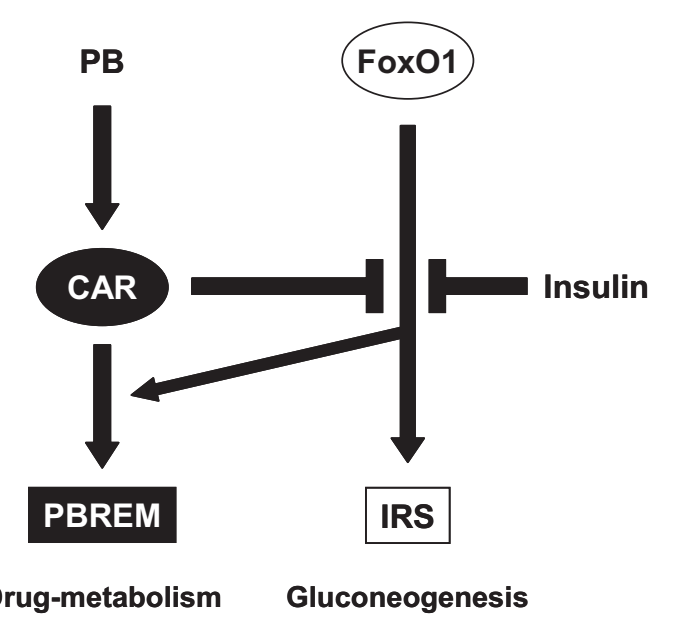

Figure 2 Schematic representation of CAR-FoxO1 cross-talk. Upon PB activation, CAR forms a complex with RXR and binds to PBREM, resulting in the induction of drug metabolizing enzymes. FoxO1 directly binds and activates IRS, up-regulating gluocneogeic enzymes. Insulin excludes FoxO1 from the nucleus, preventing binding of FoxO1 to the IRS. In addition, CAR binds directly to FoxO1, thus preventing the FoxO1-IRS interaction. Thus, both insulin and PB repress the FoxO1-IRS activity. Because FoxO1 is a co-activator of CAR, the insulindependent nuclear exclusion of FoxO1 results in repression of PB-CAR-PBREM activity. Arrows indicate activation or co-activation, while stop bars indicate repression or co-repression. 
no longer binds to the IRS, the activation of CAR results in repressing the PEPCK1 and G6Pase genes. PB treatment is known to improve insulin sensitivity in diabetic patients (Lahtela et al., 1985), which is explained by the fact that both insulin and CAR repress gluconeogenesis through regulating the same transcription factor FoxO1. Consistent with the repression of FoxO1 by CAR, after chronic treatment with $\mathrm{PB}$, serum glucose remains at normal levels in the $\mathrm{Car}^{+/+}$mice, but is elevated in the $\mathrm{Car}^{-/-}$mice. The regulation of FoxO1 by CAR is the first observation that CAR can work independently of the PBREM and bind directly to and co-regulate another transcription factor.

At the same time, when PB was first reported to induce drug metabolism in the early 1960s, scientists at the National Institute of Environmental Health Sciences found that insulin represses drug metabolism (Dixon et al., 1961). Hepatic levels of CYP2B and CYP3A were increased in diabetic mice and rats and insulin treatment decreased them to normal levels (Sakuma et al., 2001; Yamazoe et al., 1989). In support of these observations, insulin blocks PB induction of CYP2B in rat primary hepatocytes (Yoshida et al., 1996; Sidhu and Omiecinski, 1999). In addition, we demonstrated that insulin repression of the Cyp2b10 gene is mediated by FoxO1 (Kodama et al., 2004), which co-activates CAR and augments CAR-mediated PBREM activity in HepG2 cells. Insulin represses this FoxO1-dependent co-activation of CAR activity. Moreover, co-expression of constitutively activated Akt also represses CAR activity. Thus, by excluding it from the nucleus, insulin limits the availability of the co-activator FoxO1 to enhance CAR activity, resulting in the repression of CAR-PBREM activity. In addition to CAR, pregnane $X$ receptor (PXR) also co-represses FoxO1-IRS activity. Similar to CAR, FoxO1 co-represses PXR-mediated trans-activation. Thus, drug-insulin interactions can be mediated by the reciprocal regulation by CAR/PXR and FoxO1 of their specific target genes (Fig. 2).

The most severe form of adverse effect of PB exposure is the development of liver tumors in rodents. Acute treatment with PB causes hepatomegaly, an increase in cellular hypertrophy and hyperplasia (Wei et al., 2000). It was first reported in the 1970s that chronic PB treatment promotes hepatocellular carcinoma (HCC) (Peraino et al., 1971). PB is the prototype of many so-called nongenotoxic carcinogens that cause tumors without mutating DNA. Despite intense investigations for decades, the molecular mechanism of $\mathrm{PB}$ promotion of HCC remains virtually unknown. Because CAR is activated by $\mathrm{PB}$, it was logical for us to examine whether CAR is involved in PB promotion of HCC. Yukio Yamamoto studied this subject and the results were unquestionably clear: CAR is an essential factor in PB promotion of HCC (Yamamoto et al., 2004). In this study, liver tumors were initiated by a single injection of a typical genotoxic carcinogen diethylnitrosamine (DEN) in $\mathrm{Car}^{+/+}$and $\mathrm{Car}^{-/-}$mice, subjected to chronic treatment with PB. After 35 weeks of PB treatment, HCC only developed in the $\mathrm{Car}^{+/+}$mice. After 50 weeks, all the $\mathrm{Car}^{+/+}$mice had died of liver tumors, while no $\mathrm{Car}^{-/-}$mice had either developed liver tumors or died. In the absence of CAR, PB does not promote HCC.

The molecular mechanism by which CAR promotes HCC is not known. Because non-genotoxic carcinogens can both increase cell proliferation and suppress apoptosis, CAR may regulate factors (enzymes, receptors, and proteins) that are involved in the regulation of proliferation and/or apoptosis. CAR may regulate those factors through gene expressions, as well as direct protein-protein interactions. No consensus on what these factors may be has arisen yet, although c-Jun, FoxM1B, Connexin32, and Mdm2 have been suggested as candidates (Eferl et al., 2003; Kalinichenko et al., 2004; Moennikes et al., 2000, Huang et al., 2005). Although PB has been used to treat epilepsy patients for decades, liver tumors have never been associated with PB treatment, PB does not cause 
liver tumors in human. However, the role of CAR in human HCC development should not be ruled out at the moment, since there is intriguing suggestions that CAR might be involved in this. $\mathrm{C} 3 \mathrm{H}$ mice are susceptible to $\mathrm{PB}$ promotion of $\mathrm{HCC}$, while C57/BL6 mice are resistant to it. Recent genetic studies linked this susceptibility to the markers D1Mit33 of mouse chromosome 1 (Bilger et al., 2004). The corresponding region 1q21-23 of human chromosome 1 is found to be amplified in more than half of human HCC samples. Intriguingly, the Car locus is present within this region of both mouse and human chromosomes 1 . Thus, the relationship of CAR to HCC deserves intense investigations.

Mechanism of CAR activation. CAR, as its name indicates, is a constitutively activated receptor, making any effort to investigate its "activation mechanism" sound irrational. On the contrary, the fact is that this constitutive active nature is tightly controlled in liver in vivo, allowing CAR to be activated in response to PB. CAR is also characterized as a nuclear receptor that is activated without directly binding to $\mathrm{PB}$, making the investigation challenging and adventurous. The ultimate question is: What protein, lipid or carbohydrate binds PB directly and triggers CAR activation? Therefore, it is important to decipher the cellular and molecular events occurring during CAR activation. Two recent advances that address this question are described next; CAR nuclear translocation regulated by dephosphorylation and the transcriptional regulation of the $C Y P 2 B$ gene by multiple CAR binding sites.

$\mathrm{PB}$ triggers $\mathrm{CAR}$, which is sequestered in the cytoplasm, to translocate into the nucleus. Okadaic acid (OA), the serine/threonine protein phosphatase inhibitor, represses PB-triggered nuclear translocation and PB induction of the Cyp $2 b$ gene in mouse primary hepatocytes (Kawamoto et al., 1999). While OA repression suggests that dephosphorylation of CAR may be an essential step regulating the CAR translocation, previous efforts to demonstrate CAR phosphorylation always ended in disappointment. Fardin Hosseinpour has recently undertaken the laborious, yet ingenious task of identifying Ser-202 of mouse CAR as a key phosphorylation site (Fig. 3, Hosseinpour et al., 2006). Given the fact that the ligand-binding domain of mouse PXR can translocate into the nucleus in response to $\mathrm{PB}$, when it is placed after the DNA binding domain of CAR, all serine and threonine residues conserved in the ligand-binding domains of both CAR and PXR were selected and mutated to aspartic acid. These mutants were directly expressed in mouse livers to examine their nuclear translocation. Only the mutation of serine-202 to aspartic acid retained CAR in the cytoplasm after PB treatment. On the other hand, the alanine mutation of serine-202 allowed normal translocation of CAR into the nucleus. An antibody against a peptide containing phosphorylated Ser-202 was employed to show that serine-202 is phosphorylated only in the cytoplasm of HepG2 cells. Thus, serine-202 is a critical phosphorylation site in the ligand-binding domain and its dephosphorylation is required for $\mathrm{CAR}$ to undergo $\mathrm{PB}$-triggered nuclear translocation.

CAR forms a complex with Hsp90 to remain in the cytoplasm of mouse hepatocytes in vivo. This CAR:Hsp90 complex recruits protein phosphatase 2A (PP2A) in response to PB (Yoshinari et al., 2003). If PP2A is the protein phosphatase that dephosphorylates serine-202, PB should elicit a signal pathway to regulate PP2A for dephosphorylating serine-202. The question arose as to what pathway PB might elicit. Recently, PB has been reported to activate the AMP-activating protein kinase (AMPK) in HepG2-derived WGA cells that are capable of inducing $C Y P 2 B$ gene in response to PB (Rencurel et al., 2005). AMPK regulates the CAR-mediated activation of PBREM in WGA cells. AMPK is an adaptive as well as systemic energy sensor and has been implicated in obesity and type-2 diabetes (Kahn et al., 2005). Whether AMPK regulates PP2A to dephophorylate serine-202 


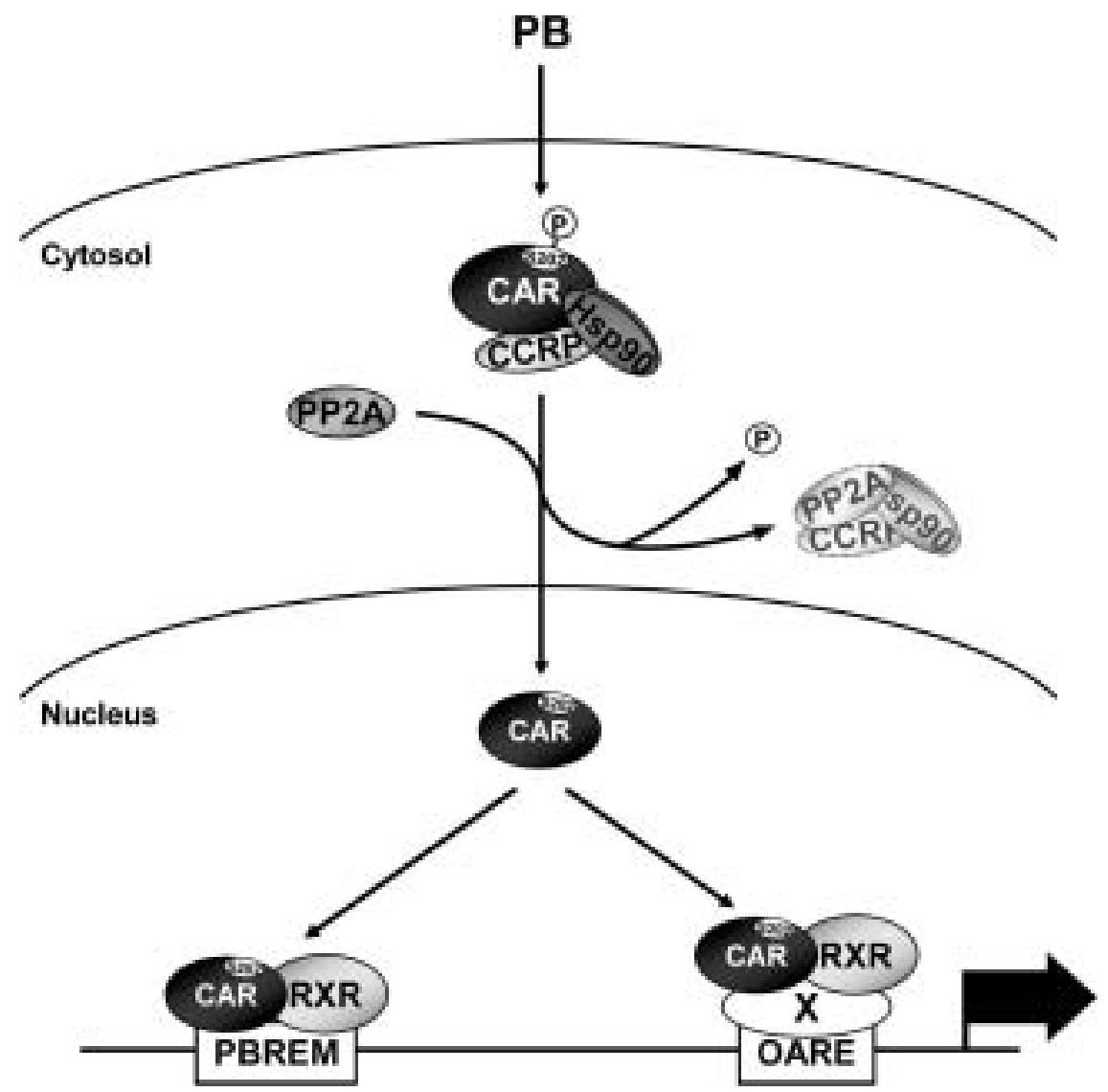

Figure 3 The "multi-tasks" mechanism of CAR activation. In the absence of PB, mouse CAR is phospholylated at Ser-202 and forms a complex with Hsp90 and CCRP in the cytoplasm. Upon activation by PB, PP2A dephosphorylates CAR, resulting in dissociation of CAR from the complex and translocation into the nucleus. CAR herodimerizes with RXR in the nucleus and interacts with the two distinct elements PBREM and OARE to synergistically activate the transcription of $C Y P 2 B 6$ gene.

remains a question for future investigations. Over-expression experiments show that the CAR:Hsp90 complex also contains a co-chaperone, known as cytoplasmic CAR retention protein, or CCRP. CCRP is a tetratricopeptide repeat protein and accumulates CAR in the cytoplasm of HepG2 cells. The possibility exists that CCRP is also involved in regulating the desphosphorylation of serine-202. In addition to this advance in the area of the nuclear translocation of CAR, we have also obtained a new insight into understanding the regulatory mechanism of CAR-mediated transcription.

Karen Swales found another example that CAR acts as co-activator to regulate transcription (Fig. 3, Swales et al., 2005). Ym17 cells are a stable HepG2 cell line that expresses V5-tagged mouse CAR in the nucleus. Tagging with the V5 peptide at the C-terminus abrogates high constitutive activity of mouse CAR in cell-based Luc reporter assays and converts it to a receptor that can be effectively activated by TCPOBOP. The endogenous CYP2B6 gene is synergistically induced by co-treatment with TCPOBOP and $\mathrm{OA}$ in the Ym17 cells. Because CAR has already accumulated in the nucleus of Ym17 cells before drug treatment, this synergistic induction should be regulated at the level of 
transcription in the nucleus. The $1.8-\mathrm{kbp} C Y P 2 B 6$ promoter is activated by TCPOBOP in the transfected Ym17 cells and is synergistically up-regulated by co-treatment with OA. The synergistic activation is regulated by the binding of CAR to two distinct sites within the $C Y P 2 B 6$ gene: the distal PBREM and proximal OA response element (OARE). Acting as the transcription factor, direct binding of CAR activates PBREM (-1732/-1685 bp) in response to TCPOBOP. OARE, which is located between -256 and -233 bp of the promoter, is responsible for the synergistic activation by OA. OARE requires CAR to acquire this synergistic activation. Both DNA affinity chromatography and chromatin immunoprecipitation assays have shown that, in fact, CAR binds to OARE after co-treatment with TCPOBOP and OA. The binding of CAR to OARE is indirect and mediated by yet an unknown DNA-binding protein. Thus, CAR interacts with the two distinct elements, PBREM and OARE, to synergistically activate the transcription of the $C Y P 2 B 6$ gene. This is a novel mechanism in which a given receptor regulates multiple distinct enhancer elements of a single gene by acting as both a transcription factor and a co-regulator. We call this mechanism the "multi-tasks" mechanism. Further characterization of the "multi tasks" mechanism of CAR-mediated transcription should greatly enhance our knowledge of nuclear receptors, their functions, and mechanisms.

\section{PROSPECTS}

By activating CAR, PB accomplishes its diverse effects on liver functions (Fig. 1). One of these effects is the coordinate induction of a large group of phase I and II drug metabolizing enzymes and transporters, through the CAR-mediated activation of PBREM found in the genes that encode those enzymes and transporters. Because both xenobiotics and endobiotics share the same enzymes and transporters for metabolism and excretion, CAR activation by $\mathrm{PB}$ results in the alteration of liver functions proactively or adversely. CAR also exerts its effects by acting as a co-regulator of gene transcription. Co-repression of FoxO1 is only the example of this type of CAR regulation at the present time. However, many situations similar to the CAR-FoxO1 cross-talk may be waiting to be discovered in future investigations, thus shedding light on drug interactions with endogenous signals such as insulin. Unlike the classic model of nuclear receptor action in which a receptor directly binds to its enhancer element, CAR regulates multiple distinct elements of a single gene. Once the molecular mechanism called "multi-tasks" is further understood, it will provide a number of targets for drug development and discovery. Our cDNA microarray study using $\mathrm{Car}^{-1-}$ mice showed more than 140 genes that are either up- or down-regulated by PB treatment, yet CAR regulates only half of those genes (Ueda et al, 2002). Thus, CAR is not the only factor mediating PB actions; it can explain, at most, half of the diverse effects of $\mathrm{PB}$. We hasten to find factors that regulate CAR-independent mechanisms for the complete understanding of PB actions.

\section{ABBREVIATIONS}

CAR constitutive active/androstane receptor

CPT1A carnitine palmitoyltransferase 1a.

PXR pregnane $X$ receptor

$\mathrm{RXR} \quad$ retinoid $\mathrm{X}$ receptor

TCPOBOP 1,4-bis[2-(3,5-dichloropyridyloxy)]benzene 


\section{REFERENCES}

Argaud, D., Halimi, S., Catelloni, F., Leverve, X. M. (1991). Inhibition of gluconeogenesis in isolated rat hepatocytes after chronic treatment with phenobarbital. Biochem. J. 280:663-669.

Assem, M., Schuetz, E. G., Leggas, M., Sun, D., Yasuda, K., Reid, G., Zelcer, N., Adachi, M., Strom, S., Evans, R. M., Moore, D. D., Borst, P., Schuetz, J. D. (2004). Interactions between hepatic Mrp4 and Sult2a as revealed by the constitutive androstane receptor and Mrp4 knockout mice. J. Biol. Chem. 279:22250-22257.

Barthel, A., Schmoll, D., Unterman, T. G. (2005). FoxO proteins in insulin action and metabolism. Trends Endocrinol. Metab. 16:183-189.

Bianco, A. C., Salvatore, D., Gereben, B., Berry, M. J., Larsen, P. R. (2002). Biochemistry, cellular and molecular biology, and physiological roles of the iodothyronine selenodeiodinases. Endocr. Rev. 23:38-89.

Biggs, W. H., 3rd, Meisenhelder, J., Hunter, T., Cavenee, W. K., Arden, K. C. (1999). Protein kinase B/Akt-mediated phosphorylation promotes nuclear exclusion of the winged helix transcription factor FKHR1. Proc. Natl. Acad. Sci. USA 96:7421-7426.

Bilger, A., Bennett, L. M., Carabeo, R. A., Chiaverotti, T. A., Dvorak, C., Liss, K. M., Schadewald, S. A., Pitot, H. C., Drinkwater, N. R. (2004). A potent modifier of liver cancer risk on distal mouse chromosome 1: linkage analysis and characterization of congenic lines. Genetics 167:859-866.

Conney, A. H. (1967). Pharmacological implication of microsomal enzyme induction. Pharmacol. Rev. 19:317-366.

Curran, P. G., DeGroot, L. J. (1991). The effect of hepatic enzyme-inducing drugs on thyroid hormones and the thyroid gland. Endocr. Rev. 12:135-150.

Diwan, B. A., Henneman, J. R., Rice, J. M., Nims, R. W. (1996). Enhancement of thyroid and hepatocarcinogenesis by 1,4-bis[2-(3,5-dichloropyridyloxy)]benzene in rats at doses that cause maximal induction of CYP2B. Carcinogenesis 17:37-43.

Dixon, R. L., Hart, L. G., Fouts, J. R. (1961). The metabolism of drugs by liver microsomes from alloxan-diabetic rats. J. Pharmacol. Exp. Ther. 133:7-11.

Eferl, R., Ricci, R., Kenner, L., Zenz, R., David, J. P., Rath, M., Wagner, E. F. (2003). Liver tumor development, c-Jun antagonizes the proapoptotic activity of p53. Cell 112:181-192.

Eloranta, J. J., Kullak-Ublick, G. A. (2005). Coordinate transcriptional regulation of bile acid homeostasis and drug metabolism. Arch. Biochem. Biophys. 433:397-412.

Guo, G. L., Lambert, G., Negishi, M., Ward, J. M., Brewer, H. B., Jr., Kliewer, S. A., Gonzalez, F. J., Sinal, C. J. (2003). Complementary roles of farnesoid $\mathrm{X}$ receptor, pregnane $\mathrm{X}$ receptor, and constitutive androstane receptor in protection against bile acid toxicity. J. Biol. Chem. 278:45062-45071.

Hegedus, L., Hansen, J. M., Luhdorf, K., Perrild, H., Feldt-Rasmussen, U., Kampmann, J. P. (1985). Increased frequency of goitre in epileptic patients on long-term phenytoin or carbamazepine treatment. Clin. Endocrinol. (Oxf) 23:423-429.

Hempel, N., Wang, H., LeCluyse, E. L., McManus, M. E., Negishi, M. (2004). The human sulfotransferase SULT1A1 gene is regulated in a synergistic manner by Sp1 and GA binding protein. Mol. Pharmacol. 66:1690-1701.

Hiasa, Y., Kitahori, Y., Ohshima, M., Fujita, T., Yuasa, T., Konishi, N., Miyashiro, A. (1982). Promoting effects of phenobarbital and barbital on development of thyroid tumors in rats treated with N-bis(2-hydroxypropyl)nitrosamine. Carcinogenesis 3:1187-1190.

Honkakoski, P., Moore, R., Washburn, K. A., Negishi, M. (1998). Activation by diverse xenochemicals of the 51-base pair phenobarbital-responsive enhancer module in the CYP2B10 gene. Mol. Pharmacol. 53:597-601.

Hosseinpour, F., Moore, R., Negishi, M., Sueyoshi, T. (2006). Ser-202 regulates the nuclear translocation of constitutive active/androstane receptor CAR. (In press). 
Huang, W., Zhang, J., Chua, S. S., Qatanani, M., Han, Y., Granata, R., Moore, D. D. (2003). Induction of bilirubin clearance by the constitutive androstane receptor (CAR). Proc. Natl. Acad. Sci. USA 100:4156-4161.

Huang, W., Zhang, J., Washington, M., Liu, J., Parant, J. M., Lozano, G., Moore, D. D. (2005). Xenobiotic stress induces hepatomegaly and liver tumors via the nuclear receptor constitutive androstane receptor. Mol. Endocrinol. 19:1646-1653.

Jenkins, J. K., Boothby, L. A. (2002). Treatment of itching associated with intrahepatic cholestasis of pregnancy. Ann. Pharmacother. 36:1462-1465.

Kahn, B. B., Alquier, T., Carling, D., Hardie, D. G. (2005). AMP-activated protein kinase: ancient energy gauge provides clues to modern understanding of metabolism. Cell Metab 1:15-25.

Kalinichenko, V. V., Major, M. L., Wang, X., Petrovic, V., Kuechle, J., Yoder, H. M., Dennewitz, M. B., Shin, B., Datta, A., Raychaudhuri, P., Costa, R. H. (2004). Foxm1b transcription factor is essential for development of hepatocellular carcinomas and is negatively regulated by the p19ARF tumor suppressor. Genes Dev. 18:830-850.

Kast, H. R., Goodwin, B., Tarr, P. T., Jones, S. A., Anisfeld, A. M., Stoltz, C. M., Tontonoz, P., Kliewer, S., Willson, T. M., Edwards, P. A. (2002). Regulation of multidrug resistanceassociated protein 2 (ABCC2) by the nuclear receptors pregnane $\mathrm{X}$ receptor, farnesoid X-activated receptor, and constitutive androstane receptor. J. Biol. Chem. 277:2908-2915.

Kawamoto, T., Sueyoshi, T., Zelko, I., Moore, R., Washburn, K., Negishi, M. (1999). Phenobarbital-responsive nuclear translocation of the receptor CAR in induction of the CYP2B gene. Mol. Cell. Biol. 19:6318-6322.

Kodama, S., Koike, C., Negishi, M., Yamamoto, Y. (2004). Nuclear receptors CAR and PXR cross talk with FOXO1 to regulate genes that encode drug-metabolizing and gluconeogenic enzymes. Mol. Cell. Biol. 24:7931-7940.

Lahtela, J. T., Arranto, A. J., Sotaniemi, E. A. (1985). Enzyme inducers improve insulin sensitivity in non-insulin-dependent diabetic subjects. Diabetes 34:911-916.

Li, X., Clemens, D. L., Anderson, R. J. (2001). Characterization of human liver thermostable phenol sulfotransferase (SULT1A1) allozymes with 3,3',5-triiodothyronine as the substrate. J. Endocrinol. 171:525-532.

Maglich, J. M., Watson, J., McMillen, P. J., Goodwin, B., Willson, T. M., Moore, J. T. (2004). The nuclear receptor CAR is a regulator of thyroid hormone metabolism during caloric restriction. J. Biol. Chem. 279:19832-19838.

Manenti, G., Dragani, T. A., Della Porta, G. (1987). Effects of phenobarbital and 1,4-bis[2-(3,5-dichloropyridyloxy)]benzene on differentiated functions in mouse liver. Chem. Biol. Interact. 64:83-92.

Matsuzaki, H., Daitoku, H., Hatta, M., Tanaka, K., Fukamizu, A. (2003). Insulin-induced phosphorylation of FKHR (Foxo1) targets to proteasomal degradation. Proc. Natl. Acad. Sci. USA 100:11285-11290.

Mizukami, Y., Sogawa, K., Suwa, Y., Muramatsu, M., Fujii-Kuriyama, Y. (1983). Gene structure of a phenobarbital-inducible cytochrome P-450 in rat liver. Proc. Natl. Acad. Sci. USA 80:3958-3962.

Moennikes, O., Buchmann, A., Romualdi, A., Ott, T., Werringloer, J., Willecke, K., Schwarz, M. (2000). Lack of phenobarbital-mediated promotion of hepatocarcinogenesis in connexin32null mice. Cancer Res. 60:5087-5091.

Nakae, J., Kitamura, T., Silver, D. L., Accili, D. (2001). The forkhead transcription factor Foxol (Fkhr) confers insulin sensitivity onto glucose-6-phosphatase expression. J. Clin. Invest. 108:1359-1367.

Negishi, M., Omura, T. (1970). Presence of apo-cytochrome 5 in microsomes from rat liver. J. Biochem. (Tokyo) 67:745-747.

Peraino, C., Fry, R. J., Staffeldt, E. (1971). Reduction and enhancement by phenobarbital of hepatocarcinogenesis induced in the rat by 2-acetylaminofluorene. Cancer Res. 31:1506-1512.

Puigserver, P., Rhee, J., Donovan, J., Walkey, C. J., Yoon, J. C., Oriente, F., Kitamura, Y., Altomonte, J., Dong, H., Accili, D., Spiegelman, B. M. (2003). Insulin-regulated hepatic gluconeogenesis through FOXO1-PGC-1alpha interaction. Nature 423:550-555. 
Qatanani, M., Zhang, J., Moore, D. D. (2005). Role of the constitutive androstane receptor in xenobiotic-induced thyroid hormone metabolism. Endocrinology 146:995-1002.

Ramsden, R., Beck, N. B., Sommer, K. M., Omiecinski, C. J. (1999). Phenobarbital responsiveness conferred by the 5'-flanking region of the rat CYP2B2 gene in transgenic mice. Gene 228:169-179.

Rena, G., Prescott, A. R., Guo, S., Cohen, P., Unterman, T. G. (2001). Roles of the forkhead in rhabdomyosarcoma (FKHR) phosphorylation sites in regulating 14-3-3 binding, transactivation and nuclear targetting. Biochem. J. 354:605-612.

Rencurel, F., Stenhouse, A., Hawley, S. A., Friedberg, T., Hardie, D. G., Sutherland, C., Wolf, C. R. (2005). AMP-activated protein kinase mediates phenobarbital induction of CYP2B gene expression in hepatocytes and a newly derived human hepatoma cell line. J. Biol. Chem. 280:4367-4373.

Sakuma, T., Honma, R., Maguchi, S., Tamaki, H., Nemoto, N. (2001). Different expression of hepatic and renal cytochrome P450s between the streptozotocin-induced diabetic mouse and rat. Xenobiotica 31:223-237.

Schmoll, D., Walker, K. S., Alessi, D. R., Grempler, R., Burchell, A., Guo, S., Walther, R., Unterman, T. G. (2000). Regulation of glucose-6-phosphatase gene expression by protein kinase Balpha and the forkhead transcription factor FKHR. Evidence for insulin response unit-dependent and -independent effects of insulin on promoter activity. J. Biol. Chem. 275:36324-36333.

Sidhu, J. S., Omiecinski, C. J. (1999). Insulin-mediated modulation of cytochrome P450 gene induction profiles in primary rat hepatocyte cultures. J. Biochem. Mol. Toxicol. 13:1-9.

Sinal, C. J., Tohkin, M., Miyata, M., Ward, J. M., Lambert, G., Gonzalez, F. J. (2000). Targeted disruption of the nuclear receptor FXR/BAR impairs bile acid and lipid homeostasis. Cell 102:731-744.

Sueyoshi, T., Kawamoto, T., Zelko, I., Honkakoski, P., Negishi, M. (1999). The repressed nuclear receptor CAR responds to phenobarbital in activating the human CYP2B6 gene. J. Biol. Chem. 274:6043-6046.

Sueyoshi, T., Negishi, M. (2001). Phenobarbital response elements of cytochrome P450 genes and nuclear receptors. Annu. Rev. Pharmacol. Toxicol. 41:123-143.

Sugatani, J., Yamakawa, K., Yoshinari, K., Machida, T., Takagi, H., Mori, M., Kakizaki, S., Sueyoshi, T., Negishi, M., Miwa, M. (2002). Identification of a defect in the UGT1A1 gene promoter and its association with hyperbilirubinemia. Biochem. Biophys. Res. Commun. 292:492-497.

Swales, K., Negishi, M. (2004). CAR, driving into the future. Mol. Endocrinol. 18:1589-1598.

Swales, K., Kakizaki, S., Yamamoto, Y., Inoue, K., Kobayashi, K., Negishi, M. (2005). Novel CARmediated mechanism for synergistic activation of two distinct elements within the human cytochrome P450 2B6 gene in HepG2 cells. J. Biol. Chem. 280:3458-3466.

Trottier, E., Belzil, A., Stoltz, C., Anderson, A. (1995). Localization of a phenobarbital-responsive element (PBRE) in the 5'-flanking region of the rat CYP2B2 gene. Gene 158:263-268.

Ueda, A., Hamadeh, H. K., Webb, H. K., Yamamoto, Y., Sueyoshi, T., Afshari, C. A., Lehmann, J. M., Negishi, M. (2002). Diverse roles of the nuclear orphan receptor CAR in regulating hepatic genes in response to phenobarbital. Mol. Pharmacol. 61:1-6.

Visser, T. J. (1990). Importance of deiodination and conjugation in the hepatic metabolism of thyroid hormone. In: Greer, M. A., ed. The Thyroid Gland. New York: Raven Press. pp. 255-283.

Wang, H., Faucette, S., Moore, R., Sueyoshi, T., Negishi, M., LeCluyse, E. (2004). Human constitutive androstane receptor mediates induction of CYP2B6 gene expression by phenytoin. J. Biol. Chem. 279:29295-29301.

Wei, P., Zhang, J., Egan-Hafley, M., Liang, S., Moore, D. D. (2000). The nuclear receptor CAR mediates specific xenobiotic induction of drug metabolism. Nature 407:920-923.

Yamamoto, Y., Kawamoto, T., Negishi, M. (2003). The role of the nuclear receptor CAR as a coordinate regulator of hepatic gene expression in defense against chemical toxicity. Arch. Biochem. Biophys. 409:207-211. 
Yamamoto, Y., Moore, R., Goldsworthy, T. L., Negishi, M., Maronpot, R. R. (2004). The orphan nuclear receptor constitutive active/androstane receptor is essential for liver tumor promotion by phenobarbital in mice. Cancer Res. 64:7197-7200.

Yamazoe, Y., Murayama, N., Shimada, M., Yamauchi, K., Kato, R. (1989). Cytochrome P450 in livers of diabetic rats: regulation by growth hormone and insulin. Arch. Biochem. Biophys. 268:567-575.

Yeagley, D., Guo, S., Unterman, T., Quinn, P. G. (2001). Gene- and activation-specific mechanisms for insulin inhibition of basal and glucocorticoid-induced insulin-like growth factor binding protein-1 and phosphoenolpyruvate carboxykinase transcription. Roles of forkhead and insulin response sequences. J. Biol. Chem. 276:33705-33710.

Yoshida, Y., Kimura, N., Oda, H., Kakinuma, A. (1996). Insulin suppresses the induction of CYP2B1 and CYP2B2 gene expression by phenobarbital in adult rat cultured hepatocytes. Biochem. Biophys. Res. Commun. 229:182-188.

Yoshinari, K., Kobayashi, K., Moore, R., Kawamoto, T., Negishi, M. (2003). Identification of the nuclear receptor CAR:HSP90 complex in mouse liver and recruitment of protein phosphatase 2A in response to phenobarbital. FEBS Lett 548:17-20.

Zhang, J., Huang, W., Qatanani, M., Evans, R. M., Moore, D. D. (2004). The constitutive androstane receptor and pregnane $\mathrm{X}$ receptor function coordinately to prevent bile acid-induced hepatotoxicity. J. Biol. Chem. 279:49517-49522. 
